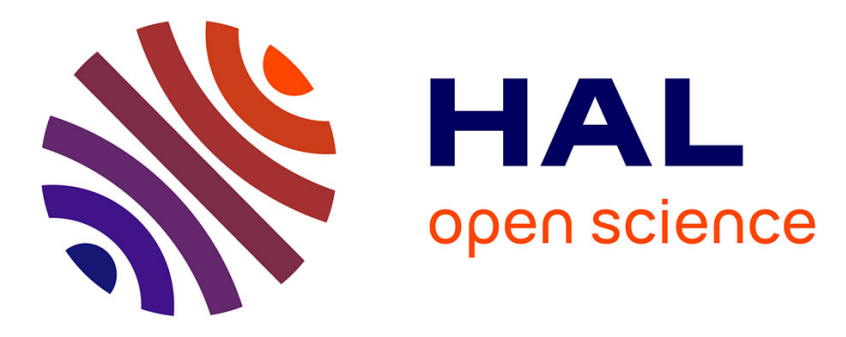

\title{
Detecting alterations in historical violins with optical monitoring
}

Alireza Rezaei, Emanuel Aldea, Piercarlo Dondi, Marco Malagodi, Sylvie Le Hégarat-Mascle

\section{- To cite this version:}

Alireza Rezaei, Emanuel Aldea, Piercarlo Dondi, Marco Malagodi, Sylvie Le Hégarat-Mascle. Detecting alterations in historical violins with optical monitoring. Fifteenth International Conference on Quality Control by Artificial Vision, May 2019, Mulhouse, France. pp.58, 10.1117/12.2521702 . hal-02190015

\section{HAL Id: hal-02190015 \\ https://hal.science/hal-02190015}

Submitted on 21 Jul 2019

HAL is a multi-disciplinary open access archive for the deposit and dissemination of scientific research documents, whether they are published or not. The documents may come from teaching and research institutions in France or abroad, or from public or private research centers.
L'archive ouverte pluridisciplinaire HAL, est destinée au dépôt et à la diffusion de documents scientifiques de niveau recherche, publiés ou non, émanant des établissements d'enseignement et de recherche français ou étrangers, des laboratoires publics ou privés. 


\title{
Detecting alterations in historical violins with optical monitoring
}

\author{
Alireza Rezaei ${ }^{1}$, Emanuel Aldea ${ }^{1}$, Piercarlo Dondi ${ }^{2,3}$, Marco Malagodi ${ }^{2,4}$, Sylvie Le \\ Hégarat-Mascle ${ }^{1}$ \\ ${ }^{1}$ SATIE Laboratory, UMR 8029, Paris Sud University, Orsay, France \\ ${ }^{2}$ CISRiC - Arvedi Laboratory of Non-Invasive Diagnostics, University of Pavia, Cremona, Italy \\ ${ }^{3}$ Department of Civil Engineering and Architecture, University of Pavia, Pavia, Italy \\ ${ }^{4}$ Department of Musicology and Cultural Heritage, University of Pavia, Cremona, Italy
}

\begin{abstract}
Some historical musical instruments are still played today, and are prone to be affected by mechanical wear of superficial varnishes in direct contact with the musicians. In a previous work, an ad-hoc monitoring plan for historical violins, that involves the use multiple non-invasive analytic techniques, achieved good performance. However, the high number of techniques is a limitation if we want to perform frequent checks. In this work, our aim is to rely only on UV induced fluorescence images for performing a fast, preliminary check and then, if a possible alteration is detected, conduct spectroscopic analyses, which are more precise but also more time consuming. In this study, we explore the a-contrario framework in order to allow for the automatic detection of significant changes in the superficial varnishes. The difficulty of detecting the changes is represented by the need to define the significance of a change, in the absence of a ground truth provided by the expert. Tests performed on samples that simulate the effect of surface alteration during time show promising results.
\end{abstract}

Keywords: A-contrario detection, UV fluorescence, historical violins, preventive conservation

\section{INTRODUCTION}

Preventive conservation is a field of research that focuses on reducing the risk of deterioration of artworks, with the final goal to minimize interventions on them. ${ }^{1}$ Historical musical instruments are a very unique kind of artifacts, since they are both preserved in museums and still played today, leading to a risk of mechanical wear of superficial varnishes in direct contact with the musicians. A previous work ${ }^{2}$ proposed an ad-hoc monitoring plan for historical instruments that involves the use of multiple non-invasive analytic techniques such as UV induced fluorescence (UVIFL or UVF) photography, colorimetric measures, XRF and FTIR spectroscopic analyses. In that study, two important violins were analyzed during a six months period: Vesuvio c.1727 (frequently played) made by Antonio Stradivari and Carlo IX c.1566 (rarely played) made by Andrea Amati. The achieved results were promising and allowed to monitor possible variations in the optical and chemical-physical properties of the varnishes. However, the high number of adopted techniques is a limitation if we intend to perform frequent checks on a larger set of instruments in order to detect the wear in the earliest possible stages. In this paper, we propose a new approach for searching the presence of variations in violins varnishes using only UVF images. UVF photography is a well-known non-invasive diagnostic technique, used in cultural heritage field, that allows to see details not perceivable using visible light. ${ }^{3,4}$ This is due to the properties of different materials used in artworks that react to UV-A light $(315-400 \mathrm{~nm})$ and re-emit radiations in the visible wavelength $(400-700 \mathrm{~nm})$, producing characteristic fluorescence colors. ${ }^{5}$ Since the UV radiation only reaches the most superficial layers of a surface, UVF photography alone cannot be conclusive, but can give us some hints about the presence of color variations due to materials modifications. Our goal is to use UVF image processing as a preliminary fast examination of the surface of a violin and then, only if a possible alteration is detected, perform spectroscopic analyses (punctual examinations, more precise but also more time consuming, that can give information about chemical elements presence and organic and inorganic compounds ${ }^{6}$ ). It is important to stress that this problem

Further author information: (Send correspondence to E.A.) E-mail: emanuel.aldea@u-psud.fr 
is different (and complementary) from monitoring the evolution of regions already worn out. ${ }^{7}$ In preventive conservation we want to detect the very early stages of an alteration even in areas where the varnishes are still present. Thus, we are interested in the detection of the smallest alterations that may occur on the surface of an instrument after a period of use.

The data input for our analysis is a change map for which the domain is a sub-region of the specimen (provided by an expert), and where change values are three-dimensional vectors defined in an adapted color space (i.e., HSV). The difficulty of detecting the changes is represented by the need to define the significance of a change, in the absence of a ground truth provided by the expert. Since it is not feasible to characterize directly the behavior of the target pixels (related to a possible alteration), the prior knowledge regarding the evolution of their appearance is very imprecise, and it is more effective to model the background data rather than to model accurately the category of interest.

In this study, we explored the a-contrario framework to allow for automatic detection of significant changes in the superficial varnishes. Since the seminal articles of Desolneux et al., ${ }^{8,9}$ detection approaches based on the Number of False Alarms criterion (NFA) became more and more popular in the field of image processing. In these approaches, the notion 'a-contrario' refers to the fact that detection is performed by contradicting a 'naive' model that represents the null hypothesis $\left(H_{0}\right)$ in statistical decision theory. By defining a measurement of deviation relatively to the naive model, the main asset of such approaches is their independence from threshold parameters, as they cast the detection as an optimization problem by maximizing the significance defined from deviation.

\section{DATA PREPROCESSING}

It is assumed that each sample in our series of violin surface photos has been acquired months apart from the others. Human errors in positioning the specimens lead to unavoidable differences in the appearance of the area of interest. Furthermore, aging equipment can produce different output from before. Therefore, it is necessary to account for these changes and to match as accurately as possible each time frame with the original image.

While the same illumination hardware setup and configuration are used during each capture, it is still possible for global or local differences to persist. To compensate the global variation problem, a robust approach which is effective as long as changes remain small, as in our case, is to equalize the average gray level pixel values of each frame, i.e. subtract the difference between average of two frames from the second frame:

$$
\bar{I}_{2}(p)=I_{2}(p)-\left[\mu\left(I_{2}\right)-\mu\left(I_{1}\right)\right]
$$

Additionally, the other components of the setup - camera, sample holder and the sample itself - may have slight movements and introduce mismatches between frames. Therefore it is necessary to perform a spatial registration step as well.

\subsection{Spatial registration}

In order to spatially match the samples, we rely on extracting and matching SIFT $^{10}$ features in the original image and also in the subsequent frames. Many approaches exist for registering deformable objects performing general transformations, ${ }^{11,12}$ however our captures have been performed on a rigid object in a controlled environment. Although some residual rotations may subsist, the transformation for each image pair may be approximated using small translation and scaling components (the proper alignment of the samples with respect to the imager is easier to perform during the capturing process). Figure 1 shows the detected SIFT features in a sample pair of images and the result of registration. The figure also illustrates pairs of matched features which are, in our case, very tightly coupled. These matched points are used in the next step to estimate a proper transformation between the two frames using a robust estimation method. ${ }^{13}$

After estimating the right transformation from the matched features and applying it to the moving image we assume that the alignment is achieved at pixel level. Although errors in registration may persist, this assumption is sufficient for the following process given the expected scale change. 


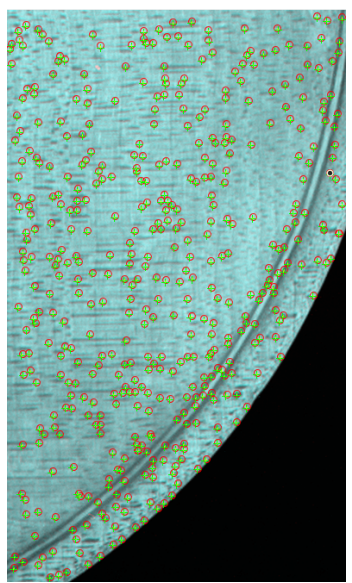

(a)

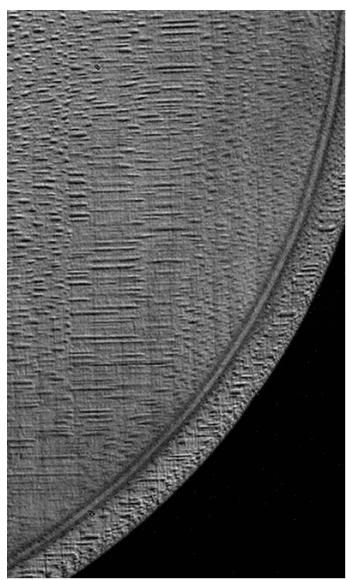

(b)

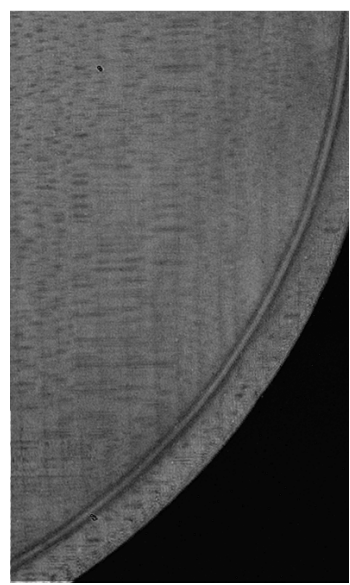

(c)

Figure 1: An example of spatial registration: (a) matched SIFT feature points; (b) difference of two frames before registration; (c) difference of two frames after registration. Brighter locations indicate higher differences.

\subsection{Difference map computation}

The previous assumption allows us to compute a difference map between each frame and the original (at time zero) frame. From now on, we will refer to the first frame as $t_{0}$ and other subsequent frames as $t_{i}$.

The difference map between $t_{0}$ and $t_{i}$ is created by computing the color difference between the corresponding pixels. We have utilized the CIEDE2000 formula ${ }^{14}$ after transforming the RGB values into the CIELAB color space. Then, the resulting values have been normalized in order to fit into the $[0,1]$ range.

It is worth noting that other approaches may also be used to create the difference map for the rest of the detection process, from a simple euclidean distance between RGB values to more complicated color difference models. ${ }^{15}$ However, in our case the employed difference provides consistent results which are in agreement with the expected wear areas.

\section{WEAR DETECTION}

In order to instantiate the a-contrario perception concept through a NFA criterion, two elements have to be defined: the 'naive' model that represents the statistics of the model to reject (the $H_{0}$ hypothesis) and the feature on which these statistics apply. Both depend on the considered data. However, since the naive model represents the absence of structure, we can choose it as representing the wide spreading of the samples, so that it will be rejected once the observations appear unlikely close with respect to the naive model.

Dealing with change detection, the decision of a change is due to the observation of a surprisingly high density of differences within local features. Such a definition can be interpreted as gathering two criteria: at pixel scale, high differences in feature images and, at region/area scale, high density of previously detected 'high differences'. In other words, we propose a two-step approach that firstly detects seeds as pixels likely to belong to a change area, and secondly detects dense areas of seeds. For each step, we use a NFA criterion to perform parameter-free decision.

\subsection{Seed detection}

Starting from the color difference image $\Delta I$ (Section 2.2) defined on the pixel domain $\mathcal{P} \subset \mathbb{N}^{2}$, we consider the naive model $\mathcal{M}_{\text {col }}$ to derive the set of seeds, called $\mathcal{S} \subseteq \mathcal{P}$, representing the pixels likely to belong to the altered areas. Specifically, denoting by $|X|$ the cardinality of a set $X$,

Definition 1 (NaIVe model $\mathcal{M}_{\text {col }}$ ). The image $\Delta I$ is a random field of $|\mathcal{P}|$ independent centered Gaussian variables $\mathcal{N}\left(0, \sigma^{2}\right)$. 
According to $\mathcal{M}_{\text {col }}$, the distribution of the sum of the squared values (SSV) on a subdomain $\mathcal{D} \subseteq \mathcal{P}$, $v_{\mathcal{D}}=\sum_{s \in \mathcal{D}}[\Delta I(s)]^{2}$, is a $\chi^{2}$ law with $|\mathcal{D}|$ degrees of freedom. Then, the probability $\mathbb{P}_{\mathcal{M}_{\text {col }}}\left(v_{\mathcal{D}}, \sigma\right)$ of observing a SSV lower than $v_{\mathcal{D}}$ by chance is given by the regularized incomplete Gamma function, and the Number of False Alarms associated to a subdomain $\mathcal{D}$ having $v_{\mathcal{D}}$ SSV is ${ }^{16,17}$

$$
N F A_{1}(\mathcal{D}, \sigma,|\mathcal{P}|)=|\mathcal{P}|\left(\begin{array}{l}
|\mathcal{P}| \\
|\mathcal{D}|
\end{array}\right) \mathbb{P}_{\mathcal{M}_{\text {col }}}\left(v_{\mathcal{D}}, \sigma\right)
$$

where $\left(\begin{array}{l}a \\ b\end{array}\right)$ is the binomial coefficient.

Then, minimizing NFA defined by Eq. (1), the result depends on the parameter $\sigma$ that controls the noise level in $\mathcal{M}_{\text {col }}$. In this case, similarly to prior work, ${ }^{16,17}$ it is computed by calculating the second moment of the image: $\sigma^{2}=\mathbb{E}(x-\mu)^{2}$ where $\mu$ is the statistical mean of the pixel values of the image $\Delta I$.

Let us $\hat{\mathcal{D}}=\operatorname{argmin}_{\mathcal{D} \subset \mathcal{P}} N F A_{1}(\mathcal{D}, \sigma,|\mathcal{P}|)$. Since the naive model represents the inconsistency in the data, $\hat{\mathcal{D}}$ is the set of pixels that are 'surprisingly' structured (with respect to the previous violin image) under the naive model assumption $\mathcal{M}_{\text {col }}$, i.e. the pixels presenting 'surprisingly' low $\Delta I$ values, so that the set of seeds $\mathcal{S}$ is the complementary of $\hat{\mathcal{D}}$ with respect to set $\mathcal{P}: \mathcal{S}=\mathcal{P} \backslash \hat{\mathcal{D}}$.

\subsection{Wear area detection}

Then, having derived $\mathcal{S}$ and represented it under the form of a binary image, we aim to detect the most significant cluster(s) of seeds. In this study, we compare two approaches: the first one assumes a parametric geometric shape of the altered areas (e.g. rectangular tiles, strips, rings, etc. like in Le Hégarat-Mascle et al. ${ }^{18}$ ), while the second approach considers a general shape clustering scheme proposed in Desolneux et al. ${ }^{9}$

In both cases, the considered naive model $\mathcal{M}_{\text {bin }}$ represents the absence of spatially consistant subset(s) of seeds. Specifically,

Definition 2 (NAive model $\mathcal{M}_{\text {bin }}$ ). The set of seeds $\mathcal{S}$ is a random set of $|\mathcal{S}|$ independent uniformly distributed variables over the image lattice $\mathcal{P}$.

Under uniform distribution model $\mathcal{M}_{b i n}$, denoting by $p_{O}$, the prior probability that a seed belongs to a parametric object $O$, the probability $\mathbb{P}_{\mathcal{M}_{\text {bin }}}\left(p_{O},|\mathcal{S}|, \kappa\right)$ of observing $\kappa$ seeds within $o$ by chance is given by the tail of the binomial distribution, and the Number of False Alarms ${ }^{9}$ is

$$
N F A_{2}\left(p_{O},|\mathcal{S}|, \kappa\right)=N_{\text {test }} \sum_{i=\kappa}^{|\mathcal{S}|}\left(\begin{array}{c}
|\mathcal{S}| \\
i
\end{array}\right) p_{O}^{i}\left(1-p_{O}\right)^{|\mathcal{S}|-i}
$$

In the previous equation, $p_{O}$ is estimated by the ratio between the area of object $O$ with respect to the whole image area. Note the slight difference with a NFA like in ${ }^{19}$ derived assuming a Bernoulli distribution of parameter $p$ for pixel binary values, so that the probability to have a given number $\kappa$ of seeds among a given number $\sharp O$ of pixels is a Binomial distribution of parameter $p$ and $N F A_{2}(p, \sharp O, \kappa)=N_{\text {test }} \sum_{i=\kappa}^{\sharp O}\left(\begin{array}{c}\sharp O \\ i\end{array}\right) p^{i}(1-p)^{\sharp O-i}$, with $p$ approximated by the ratio between the seed and the pixel numbers and $\sharp O$ the pixel number of object $O$.

In the case of a clustering approach, instead of constraining the object in terms of parametric form, a thick low resolution curve free of any seed and surrounding the object is required. Thus, denoting by $\mathcal{C}$ a cluster, its relative area $a(\mathcal{C})$ with respect to the whole image area is also the probability of a seed to belong to $\mathcal{C}$ under the naive model $\mathcal{M}_{\text {bin }}$, whereas the probability of a seed not to belong to $\mathcal{C}$ is $1-a(\mathcal{C})-a(\delta \mathcal{C})$, where $a(\delta \mathcal{C})$ is the relative area of the empty thick low resolution contour surrounding $\mathcal{C}$. Transposing Desolneux' formula ${ }^{9}$ with our notations,

$$
N F A_{2}(|\mathcal{C}|,|\mathcal{S}|, a(\mathcal{C}), a(\delta \mathcal{C}))=M_{\text {test }} \sum_{i=|\mathcal{C}|}^{|\mathcal{S}|}\left(\begin{array}{c}
|\mathcal{S}| \\
i
\end{array}\right)[a(\mathcal{C})]^{i}[1-a(\mathcal{C})-a(\delta \mathcal{C})]^{|\mathcal{S}|-i}
$$

In Eq. (2) and (3), $N_{\text {test }}$ and $M_{\text {test }}$ are the numbers of tests that controls the average number of false alarms. ${ }^{20}$ Conversely to the case of the first NFA (cf. Section 3.1), here, like in Desolneux et al., ${ }^{9}$ we take these numbers constant for a given image, i.e. independent of $O$ of $\mathcal{C}$, so that it is not involved in NFA minimization. 


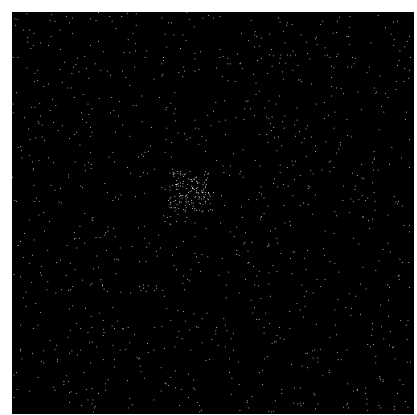

(a) A synthetic input example

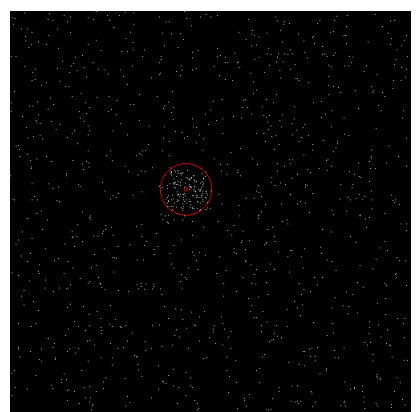

(b) Circular area detection

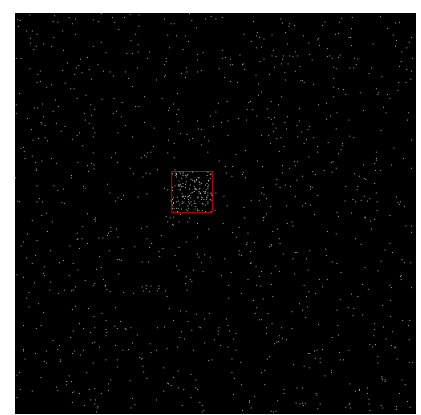

(c) Rectangular area detection

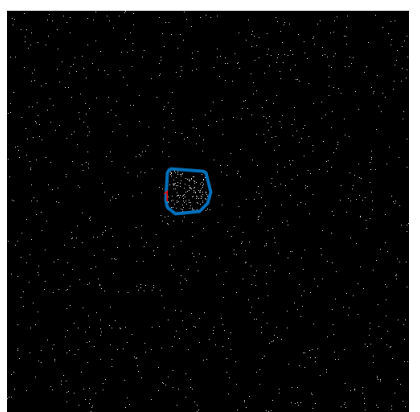

(d) Cluster detection

Figure 2: A test run for change area detection: (a) a noisy, synthetic image used as input; (b) shape constrained change detection (circular area); (c) shape constrained change detection (rectangular area); (d) cluster density based area detection.

Numerically, each cluster $\mathcal{C}$ is formed by traversing the minimum spanning tree created from the seed points $\mathcal{S}$. Then using Eq. 3, for each cluster we compute the meaningfulness(equation 4). Finally, the wear areas are separate clusters with maximum meaningfulness.

$$
S(|\mathcal{C}|,|\mathcal{S}|, a(\mathcal{C}), a(\delta \mathcal{C}))=-\log \left(N F A_{2}(|\mathcal{C}|,|\mathcal{S}|, a(\mathcal{C}), a(\delta \mathcal{C}))\right)
$$

Figure 2 illustrates a simple synthetic example. A noisy binary image with a patch of higher density noise is given to the algorithm as an input. The higher density patch has been recognized as a meaningful cluster.

\subsection{Implementation considerations}

Assuming that each frame $t_{i}$ have around $250 k$ pixels, computing and minimizing the first NFA (Eq. 1) is a demanding process. We have used special 20 digit numbers to have enough precision for this computation. Furthermore, for the minimization process it is more efficient to compute the $\log (N F A)$. Also, for the second NFA (Eq. 2), we have used the Hoeffding approximation ${ }^{21}$ in order to compute the logarithm more efficiently:

$$
\left.-\log \left(N F A_{2}((|\mathcal{C}|,|\mathcal{S}|, a(\mathcal{C}), a(\delta \mathcal{C})))\right)\right) \approx|\mathcal{S}|\left[\frac{|\mathcal{C}|}{|\mathcal{S}|} \log \left(\frac{|\mathcal{C}|}{|\mathcal{S}| * a(\mathcal{C})}\right)+\left(1-\frac{|\mathcal{C}|}{|\mathcal{S}|}\right) \log \left(\frac{1-|\mathcal{C}| /|\mathcal{S}|}{1-a(\mathcal{C})-a(\delta \mathcal{C})}\right)\right]
$$

In case of unacceptable performance problems, it is possible to resize the difference map to a more manageable size. In our case, an image of roughly $250 \mathrm{k}$ pixels gave us acceptable running time for the algorithm, more precisely approximately 20 seconds for the seed detection and 30 seconds for the clustering on non-optimized MATLAB implementations.

\section{EXPERIMENTAL RESULTS}

\subsection{Construction of a gradual wear dataset}

Since, at the moment, we have only few multi-temporal images of historical violins (further acquisitions will require several months to be significant), we created and artificially worn out a sample in laboratory for increasing our test dataset. First, the wooden sample was varnished by a violin maker in the same way adopted for a violin. Then, for simulating the effect of mechanical wear and of sweat (the most common sources of alteration), we scrubbed the sample surface using a cloth with a single drop of alcohol on it. The process was repeated twenty times to mimic the effect of a gradual alteration during a long period of time. At each step we took a UVF photo of the sample using the same protocol that we adopt for violins. ${ }^{22}$ Only the left side of the sample was altered, maintaining the right side intact as reference. A series of UVF photos of the unaltered wood sample was taken before to start the alteration to record the initial conditions. 


\subsection{Wear detection using constrained shape primitives}

Detection with shape primitives (in our case tiles and rings) has been performed on the 20 images of the altered sample when they are compared to the original unaltered surface. Figure 3 shows the result of the detection on four of these frames $\left(t_{1}, t_{6}, t_{9}\right.$ and $\left.t_{15}\right)$, selected in order to highlight the performance on various levels of wear. In each case, significant clusters have been identified using rectangular tiles or circular disks and highlighted with a bounding rectangle or circle respectively.

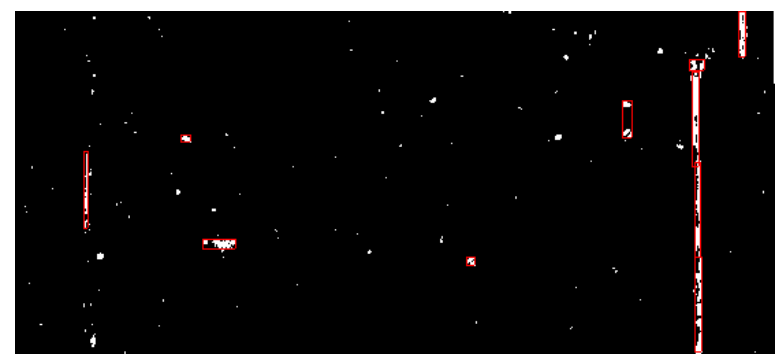

(a) $t_{1}$

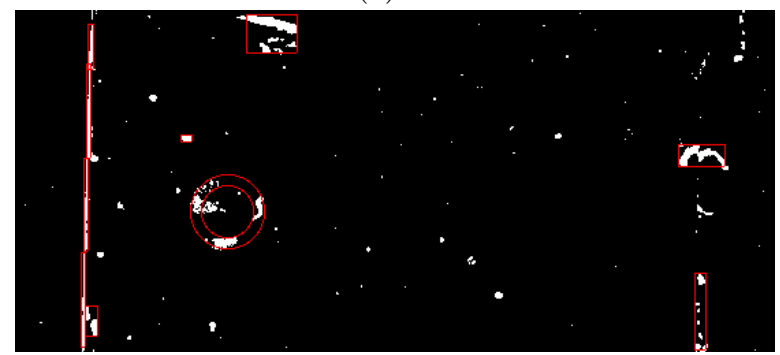

(c) $t_{9}$

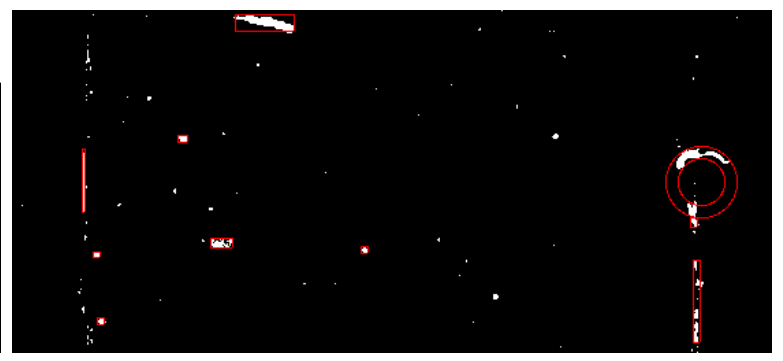

(b) $t_{6}$

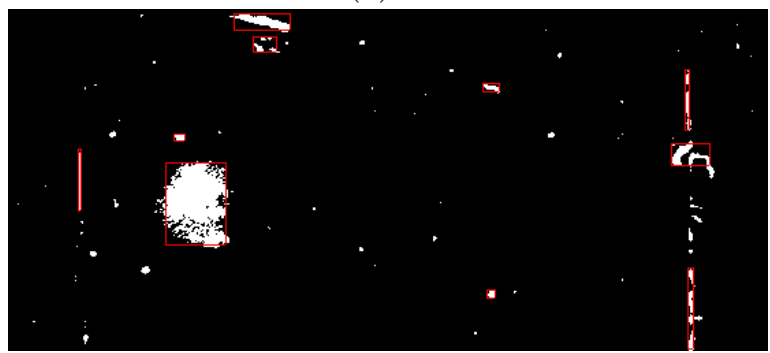

(d) $t_{15}$

Figure 3: Result of the tile and circular detection in each frame $t_{i}$ when compared to $t_{0}$.

\subsection{Wear detection using clustering}

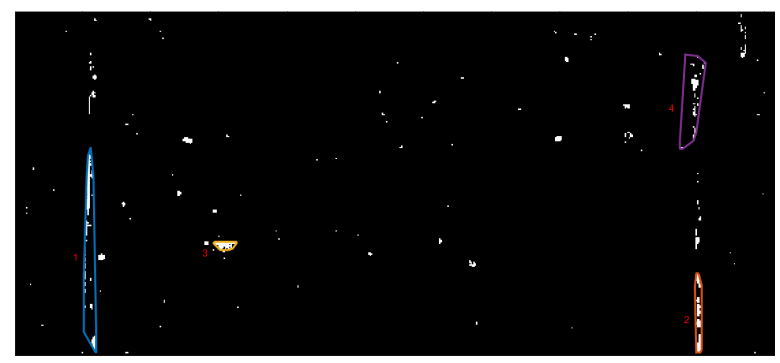

(a) $t_{1}$

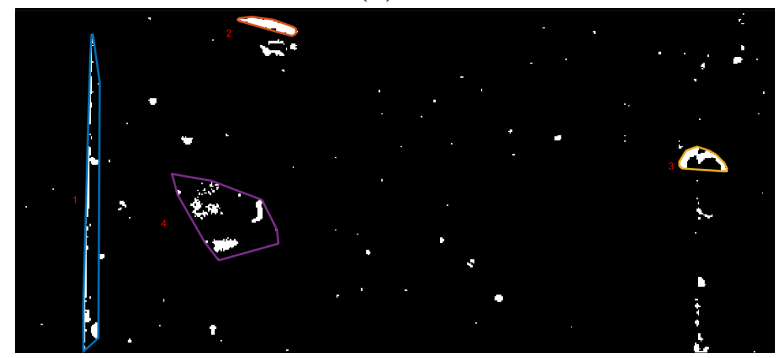

(c) $t_{9}$

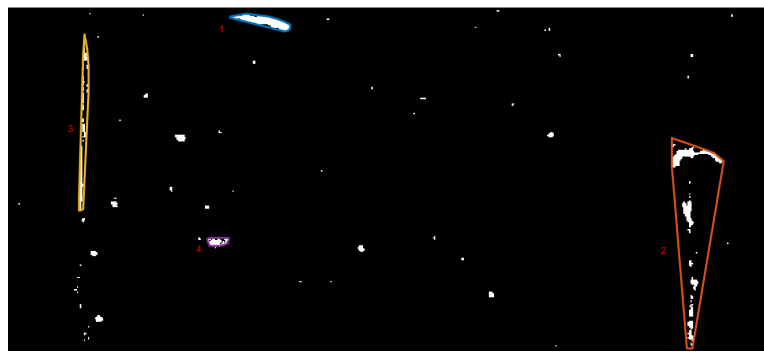

(b) $t_{6}$

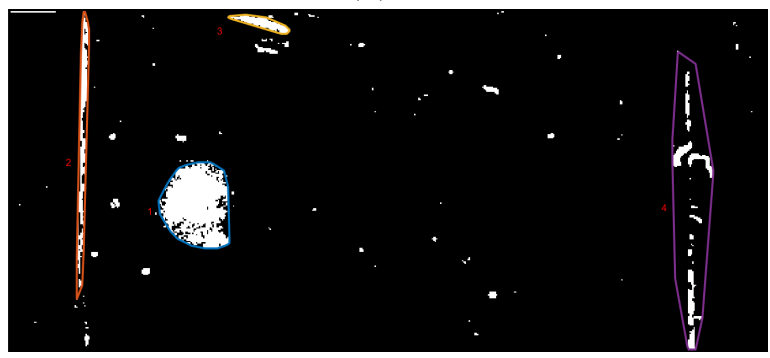

(d) $t_{15}$

Figure 4: Four most meaningful clusters in each frame $t_{i}$ when compared to $t_{0}$. 
We have also applied the cluster detection process discussed in Sections 3.1 and 3.2 on all 20 images in the dataset. In each case, we have chosen four clusters based on the highest meaningfulness values (Eq. 4). Figure 4 shows the result of the algorithm for four of these frames: $t_{1}, t_{6}, t_{9}$ and $t_{15}$. In each image, the clusters have been identified by a loose contour and the ranking of the cluster is shown by the number beside it. As it is evident, all major cluster have been correctly detected. Also, it is clear that the detection have completely ignored the noise and smaller artifacts appearing in the image.

As mentioned in Section 4.1, in both detection methods we are mainly interested in the left side of the sample, as it is the area where the change in varnish should appear. All four frames show signs of change in that area and as expected, this area keeps growing in subsequent frames. It is worth mentioning that although other relatively big clusters have been detected, they will not be considered as a worn out area since their area is constant across multiple frames. These change regions occur mainly due to the reflected UV light from the edges of the sample or unvarnished areas.

\subsection{Discussion}

The solution we propose aims to perform in a automated fashion a low-level detection, followed by a higher-level search for a pattern bearing evidence for the instrument wear. While the interest for the first step presented in Section 3.1 is clear in light of the very fine difference values observed as the wear progresses, for the second detection (Section 3.2) there is clearly a trade-off between the computational cost and the expressiveness of the pattern. Irrespective of the target configuration to be detected, we underline the fact that one of the advantages of this approach is to account for wear cues across a sub-domain of the image without relying necessarily on a strong connectivity prior of the seeds creating the pattern.

In terms of computational cost, the cluster detection process treats each seed as an independent point with respect to the others. Since in our application, the changed cluster will most likely be a dense grouping of points, it would be beneficial to consider each connected component as an individual node in the minimum spanning tree employed by the clustering algorithm. This way, we could decrease the number of clusters to check and benefit overall from a more efficient process.

\section{CONCLUSION}

In this work we studied the problem of temporal monitoring for the presence of alterations in the superficial varnishes of historical violins, using only UVF images. Our goal is to assess the feasibility of a quantitative solution for an early detection of alterations in the absence of ground truth data provided by experts. Extensive testing performed on samples that simulate the effect of surface alteration during time show that the detection process may be automated and that it is able to pinpoint changed areas from early stages of wear.

For future work, we intend to study avenues for improving the computational cost of the seed clustering. Besides, we aim to extend the seed detection process (Section 3.1) in order to assist as well with making a

decision between various color difference approaches for creating the difference map. The same process can also help in finetuning the spatial and radiometric registrations.

\section{ACKNOWLEDGMENTS}

We would like to thank "Fondazione Museo del Violino Antonio Stradivari", "Friends of Stradivari", and "Distretto Culturale di Cremona" for their collaboration. This work was partially granted by "Fondazione ArvediBuschini" of Cremona.

\section{REFERENCES}

[1] Bradley, S., "Preventive conservation research and practice at the british museum," Journal of the American Institute for Conservation 44(3), 159-173 (2005).

[2] Fichera, G. V., Albano, M., Fiocco, G., Invernizzi, C., Licchelli, M., Malagodi, M., and Rovetta, T., "Innovative monitoring plan for the preventive conservation of historical musical instruments," Studies in Conservation 63(sup1), 351-354 (2018). 
[3] Janssens, K. and Van Grieken, R., [Non-destructive micro analysis of cultural heritage materials], vol. 42, Elsevier (2004).

[4] Dondi, P., Lombardi, L., Invernizzi, C., Rovetta, T., Malagodi, M., and Licchelli, M., "Automatic analysis of uv-induced fluorescence imagery of historical violins," J. Comput. Cult. Herit. 10(2), 12:1-12:13 (2017).

[5] Stuart, B. H., [Analytical techniques in materials conservation], John Wiley \& Sons (2007).

[6] Rovetta, T., Invernizzi, C., Fiocco, G., Albano, M., Licchelli, M., Gulmini, M., Alf, G., Fabbri, D., Rombol, A., and Malagodi, M., "The case of antonio stradivari 1718 ex-san lorenzo violin: History, restorations and conservation perspectives," Journal of Archaeological Science: Reports 23, 443-450 (2019).

[7] Dondi, P., Lombardi, L., Malagodi, M., and Licchelli, M., "Automatic identification of varnish wear on historical instruments: The case of antonio stradivari violins," J. Cult. Heritage 22, 968-973 (2016).

[8] Desolneux, A., Moisan, L., and Morel, J.-M., "Meaningful alignments," IJCV 40(1), 7-23 (2000).

[9] Desolneux, A., Moisan, L., and Morel, J.-M., "A grouping principle and four applications," IEEE TPAMI 25(4), 508-513 (2003).

[10] Lowe, D. G., "Distinctive image features from scale-invariant keypoints," Int. J. Comp. Vis. 60(2), 91-110 (2004).

[11] Wu, G., Kim, M., Wang, Q., Munsell, B. C., and Shen, D., "Scalable high-performance image registration framework by unsupervised deep feature representations learning," IEEE Transactions on Biomedical Engineering 63(7), 1505-1516 (2016).

[12] de Vos, B. D., Berendsen, F. F., Viergever, M. A., Staring, M., and Išgum, I., "End-to-end unsupervised deformable image registration with a convolutional neural network," in [Deep Learning in Medical Image Analysis and Multimodal Learning for Clinical Decision Support], 204-212, Springer (2017).

[13] Torr, P. H. and Zisserman, A., "Mlesac: A new robust estimator with application to estimating image geometry," Computer vision and image understanding 78(1), 138-156 (2000).

[14] Luo, M. R., Cui, G., and Rigg, B., "The development of the cie 2000 colour-difference formula: Ciede2000," Color Research 83 Application: Endorsed by Inter-Society Color Council, The Colour Group (Great Britain), Canadian Society for Color, Color Science Association of Japan, Dutch Society for the Study of Color, The Swedish Colour Centre Foundation, Colour Society of Australia, Centre Français de la Couleur 26(5), 340350 (2001).

[15] Robertson, A. R., "The cie 1976 color-difference formulae," Color Research \& Application 2(1), 7-11 (1977).

[16] Robin, A., Moisan, L., and Le Hégarat-Mascle, S., "An a-contrario approach for subpixel change detection in satellite imagery," IEEE TPAMI 32(11), 1977-1993 (2010).

[17] Aldea, E. and Le Hégarat-Mascle, S., "Robust crack detection for unmanned aerial vehicles inspection in an a-contrario decision framework," Journal of Electronic Imaging 24(6), 061119-061119 (2015).

[18] Le Hégarat-Mascle, S., Aldea, E., and Vandoni, J., "Efficient evaluation of the number of false alarm criterion," EURASIP Journal on Image and Video Processing 2019, 35 (Feb 2019).

[19] Dibos, F., Pelletier, S., and Koepfler, G., "Real-time segmentation of moving objects in a video sequence by a contrario detection," in [Image Processing, 2005. ICIP 2005. IEEE International Conference on], $\mathbf{1}$, I-1065 (2005).

[20] Desolneux, A., Moisan, L., and Morel, J.-M., [From gestalt theory to image analysis: a probabilistic approach], vol. 34, Springer Science \& Business Media (2007).

[21] Dibos, F., Koepfler, G., and Pelletier, S., "Fast detecting and tracking of moving objects in video scenes," (2006).

[22] Dondi, P., Lombardi, L., Malagodi, M., Licchelli, M., Rovetta, T., and Invernizzi, C., "An interactive tool for speed up the analysis of UV images of Stradivari violins," in [New Trends in Image Analysis and Processing - ICIAP 2015 Workshops], Lecture Notes in Computer Science 9281, 103-110, Springer International Publishing (September 2015). 